\title{
Effect of Botulinum Toxin Injection and Physical Therapy to Reduce Tongue Pain and Discomfort: Case Reports
}

\author{
Dae-Kyung Kwon, Hee-Kyung Park \\ Department of Oral Medicine and Oral Diagnosis, School of Dentistry and Dental Research Institute, \\ Seoul National University, Seoul, Korea
}

Received December 8, 2020

Revised December 17, 2020

Accepted December 18, 2020

\section{Correspondence to:}

Hee-Kyung Park

Department of Oral Medicine and Oral

Diagnosis, Seoul National University Dental Hospital, Seoul National University School of

Dentistry, 101 Daehak-ro, Jongno-gu, Seoul 03080, Korea

Tel: +82-2-2072-2610

Fax: +82-2-744-9135

E-mail: dentopark@snu.ac.kr

https://orcid.org/0000-0003-4388-0338
The causes of tongue pain and discomfort include systemic disease, malnutrition, mental illness, fungal infection, and neuropathy. Three postmenopausal women reported burning sensations and stiffness of the tongue for various periods, from one month to four years. There were no objective etiological factors to cause the tongue pain and discomfort. Muscular tenderness upon palpation of masticatory muscles, sternocleidomastoid, trapezius, and tongue were observed. Physical therapy approaches such as moist hot pack, ultrasound, and myomonitor were performed on three patients with tongue pain, just as for temporomandibular joint disease. Additional botulinum toxin injection therapy was applied to one patient who displayed a clenching habit. All three patients showed a marked improvement in their tongue symptoms after the muscle relaxation and botulinum toxin injection therapy.

Key Words: Botulinum toxin; Burning mouth syndrome; Masticatory muscle; Physical therapy modalities; Tongue

\section{INTRODUCTION}

Various signs and symptoms of diseases are seen in the tongue, and local factors, systemic factors, and mental factors appear to be involved [1]. Burning mouth syndrome (BMS), is characterized by burning or dysaesthetic sensations or pain, often combined by taste alterations in the oral cavity without evident oral and systemic pathology, that last for more than three months [2]. Various treatment modalities for BMS were introduced from oral appliance to neuropathic medication $[1,3,4]$. Scala et al. [5] suggested that tongue discomfort resulting from identifiable precipitating factors should be classified as secondary BMS. In addition, they considered masticatory muscle disorder as a local precipitating factor of secondary BMS, and suggested that management of muscular tension/pain through physical relaxation training and physical therapy were necessary.
Siqueira et al. [6] found that the intensity of pain was positively correlated with masticatory discomfort in patients with neuropathic orofacial pain such as BMS. In addition, they suggested that even in apparent neuropathic conditions such as BMS, musculoskeletal impairment might be responsible for part of the pain and should be treated.

This study presented three cases that involved tongue pain and discomfort without specific abnormalities in the oral cavity, except tenderness on masticatory, and tongue muscles, which were reduced by muscle relaxation and botulinum toxin injection therapy. This study was approved by the Institutional Review Board of School of Dentistry at Seoul National University (S-D20200052). 


\section{CASE REPORT}

\section{Case 1}

A 71-year-old female patient visited the clinic. Her chief report was burning pain and astringent sensations in the tongue, which started around eight months ago with no apparent trigger. The numeric rating scale (NRS) of discomfort at the first visit was 5 . High blood pressure and diabetes had been well controlled since 2013. Tenderness upon palpation in all masticatory, cervical, and tongue muscles was observed. No other parafunctional habits, like clenching and bruxism, were observed. The NRS assessment remained the same when the patient revisited three weeks after selfcontrol exercise and oral lubricant use.

Moist hot pack therapy, ultrasound therapy $\left(0.7 \mathrm{~W} / \mathrm{cm}^{2}\right.$, $3 \mathrm{MHz}, 5 \mathrm{~min}$ per muscle), transcutaneous electric nerve stimulation therapy and a $6 \times 6$ exercise program for the masticatory and cervical muscles were initiated. After five weeks, the patient's NRS increased to 7. However, whenever she applied a moist heat pack, she felt the tongue pain was relieved; thus, the physical therapy and posture practice were maintained. At the follow-up after two months, the patient's NRS was reduced to 1 , and the patient showed a clear improvement in burning sensations of the tongue.

\section{Case 2}

A 61-year-old female patient visited the clinic and reported a burning sensation and stiffness in the left side of her tongue. The pain started about a month ago with no apparent trigger, and the NRS at the first visit was 9. The patient had no relevant past medical history. There were no specific findings associated with BMS except for tenderness upon palpation of all masticatory and cervical muscles, including the tongue itself. Physical therapy with the same protocol as described for the first patient was applied. After two weeks, the patient experienced a considerable reduction in symptoms and the NRS was decreased to 3. She visited the physical therapy clinic once a week and applied moist hot packs at home three to four times a week. After six weeks, the symptoms were reevaluated, and the NRS was further decreased to 1 . As her symptoms were stable, she maintained a physical therapy protocol as previously performed. The NRS score was still 1 at the three-month follow-up. She managed her tongue pain by visiting a physical therapy clinic whenever she felt tongue discomfort. The duration of the burning sensation of the tongue was a little short for BMS criteria, but physical therapy reduced the tongue symptom NRS score from 9 to 1 .

\section{Case 3}

A 60-year-old female patient visited the clinic. Her chief report was a burning and dry sensation of the mouth that started four years ago. Around a year ago, she was diagnosed with BMS at another clinic. At the first visit, the patient's NRS was 6. Topical clonazepam therapy was prescribed for three weeks. After three weeks, the patient's NRS was decreased to 2, and symptoms were significantly alleviated while on clonazepam therapy. After three months, the NRS was maintained at around 2. The patient decided to take clonazepam when needed and did not make additional appointments.

However, 21 months later, the patient visited the clinic again with the same symptoms as before, and the NRS was 5 . The patient was dissatisfied with the temporary pain relief while on topical clonazepam. She showed tenderness upon palpation of the tongue, both masseters, and left temporal muscles. Further, a clenching habit that the patient did not originally recognize was observed, even though masseter hypertrophy was not obvious. Without medication, the patient received physical therapy and an additional 60 units of botulinum toxin (clostridium botulinum toxin type A, Meditoxin inj; Medytox, Seoul, Korea) injection in both masseter muscles and 40 units in both temporal muscles. At follow up three weeks later, the burning sensation in the mouth was decreased, and the NRS was decreased to 2.

\section{DISCUSSION}

For all three patients, we performed a routine diagnostic procedure, including: clinical examination, dental radiography, clinical laboratory examination, whole salivary flow rate test, candida culture tests, the Symptom Checklist90-Revised test to exclude factors that might have caused BMS. Patients were all postmenopausal women. All patients showed no specific findings associated with secondary BMS and temporomandibular joint osteoarthritis. In addition, 
palpation of masticatory, sternocleidomastoid (SCM), and trapezius muscles was evaluated by applying a force equal to $1 \mathrm{~kg}$ for two seconds. The tongue was palpated using the thumb and index finger vertically and horizontally. All three patients showed tenderness upon palpation of these muscles. The symptom duration in case 2 was a month which was not enough to diagnose BMS. Patients in cases 1 and 2 did not receive any pharmacotherapy for BMS other than physical therapy.

Several studies have reported an association between BMS and the masticatory muscles. Corsalini et al. [7] reported that $72.7 \%$ of patients with BMS showed oral parafunctional habits, and 50\% of patients showed tenderness on palpation of the masticatory muscles; there was a statistically significant association between temporomandibular disorders (TMDs) and BMS. Yoon and Ryu [8] reported that around $85.4 \%$ of patients with BMS showed tenderness on masticatory muscle palpation. Takahashi et al. [9] reported that pushing or placing the tongue on the lower teeth may increase masticatory muscle activity in a study using electromyography. Moreover, the stress response resulting from tongue pain may increase masticatory muscle activity [7].

Studies that focused on the mechanisms of masticatory muscles contributing to BMS can be divided into several perspectives.

The first point of view is that oral parafunctional habits that involve hyperactive masticatory muscles result in repetitive microtrauma to the tongue, causing substantial neuropathic changes. Patients with bruxism, clenching, etc. often push their tongue towards the teeth [10]. Typical oral parafunctional habits related to masticatory muscle hyperactivity, such as clenching or bruxism, result in repetitive microtrauma to the tongue. Oral parafunctional habit control and a topical lubricant produced a significant improvement in burning sensations [11]. Parafunctional behavior in BMS patients is common; however, it often tends to be underestimated [12]. The patient in Case 3 had a clenching habit, and physical therapy and additional botulinum toxin injection therapy was as effective as topical clonazepam in terms of pain reduction. It seemed that control of hyperactive masticatory muscle by muscle relaxation reduced the burning and dry sensation of tongue. But the neuropathic change theory of tongue by repetitive microtrauma and its recovery mechanism needs to be defined.

The second point of view is that tongue pain and discomfort can be caused by nerve entrapment, where hyperactive masticatory muscles compress the sensory branches of the lingual nerve [13]. Isberg et al. [13] confirmed the hypothesis that lingual nerve entrapment occurs in the lateral pterygoid muscle by dissecting 52 specimens from 26 cadavers. In these specimens, the lingual nerve passed through the inferior belly of the lateral pterygoid muscle. The hyperactive masticatory muscles can compress the lingual nerve, and this lingual nerve entrapment can cause numbness, paresthesia, hypoesthesia, anesthesia, loss of taste, and pain in the tongue [14]. Alvira-González and Gay-Escoda [15] reported a case of symptom relief through masticatory muscle relaxation therapy that included physical therapy and splint therapy, among patients with a diagnosis of lingual nerve entrapment who reported pain and paresthesia in the temporal muscles and tongue for which no clinical cause was observed.

The third point of view is that referred pain induced by the trigger points of the masticatory muscles can directly cause discomfort in the tongue. Fricton et al. [16] analyzed the pattern of pain and location of trigger points in 164 patients diagnosed with myofascial pain syndrome (MPS) of the head and neck. In this study, 12.2\% of patients complained of pain in the oral cavity, tongue, and palate, and trigger points were observed in the medial pterygoid and digastric muscles. The common pain descriptors of the patients were pressure (47.5\%) and dull pain (27.4\%); however, other pain patterns that included throbbing (25.6\%), sharp (18.3\%), burning (15.9\%), and heavy (14.0\%) were also common. Donnelly et al. [17] stated that the medial pterygoid muscle and anterior belly of the digastric muscle can cause referred pain to the tongue. Hara and Namekawa [18] reported that a patient, whose cause of tongue pain could not be identified following examinations in various medical departments, was eventually diagnosed as having referred pain due to MPS of the masticatory muscles, and the pain was relieved through massage therapy and posture therapy. In addition, a central excitatory effect caused by hyperactive masticatory muscles is associated with referred pain to nearby teeth and facial areas. It can increase the discomfort of patients with BMS. BMS patients can have 
secondary musculoskeletal pain that requires treatment for the total alleviation of pain [6].

In our cases, there was tenderness in the masticatory muscle, tongue, SCM, and trapezius, even though the tongue discomforts were not induced by trigger points of the masticatory muscles. If there is a pain of the tongue muscle as well as the masticatory muscle by palpation, muscle relation therapy and parafunctional habit control in advance could be helpful before the pharmacotherapy starts. It is uncertain how much secondary musculoskeletal pain contributed to the tongue pain of these three patients. There is no consensus regarding the examination method to evaluate the contribution of the head and neck muscles to oral burning sensations. But muscle examination using the Diagnostic Criteria for TMD can be easily adapted. Additionally, the tongue muscle examination by digital palpation can be included. There are several limitations in verifying the effectiveness of the physical therapy used to reduce tongue pain and discomfort. As the interval and number of physical therapies each patient received were different, the recall interval was not consistent. Therefore, further retrospective and case control studies are needed.

\section{CONFLICT OF INTEREST}

No potential conflict of interest relevant to this article was reported.

\section{ORCID}

\author{
Dae-Kyung Kwon \\ https://orcid.org/0000-0003-3333-4472 \\ Hee-Kyung Park \\ https://orcid.org/0000-0003-4388-0338
}

\section{REFERENCES}

1. Bender SD. Burning mouth syndrome. Dent Clin North Am 2018; 62:585-596.

2. International classification of orofacial pain, 1st edition (ICOP).
Cephalalgia 2020;40:129-221.

3. Roh BY, Ahn JM, Yoon CL, Ryu JW. Case report: Treatment of burning mouth syndrome using a removable anti-nociceptive appliance. J Oral Med Pain 2012;37:1-7.

4. Grushka M, Epstein JB, Gorsky M. Burning mouth syndrome. Am Fam Physician 2002;65:615-620.

5. Scala A, Checchi L, Montevecchi M, Marini I, Giamberardino MA. Update on burning mouth syndrome: overview and patient management. Crit Rev Oral Biol Med 2003;14:275-291.

6. de Siqueira SR, Teixeira MJ, de Siqueira JT. Orofacial pain and sensory characteristics of chronic patients compared with controls. Oral Surg Oral Med Oral Pathol Oral Radiol 2013;115:e37e45.

7. Corsalini M, Di Venere D, Pettini F, Lauritano D, Petruzzi M. Temporomandibular disorders in burning mouth syndrome patients: an observational study. Int J Med Sci 2013;10:1784-1789.

8. Yoon AH, Ryu JW. Masticatory muscle tenderness in burning mouth syndrome: a case control study. Oral Biol Res 2019;43:8387.

9. Takahashi S, Kuribayashi G, Ono T, Ishiwata Y, Kuroda T. Modulation of masticatory muscle activity by tongue position. Angle Orthod 2005;75:35-39.

10. Maltsman-Tseikhin A, Moricca P, Niv D. Burning mouth syndrome: will better understanding yield better management? Pain Pract 2007;7:151-162.

11. Kho HS, Lee JS, Lee EJ, Lee JY. The effects of parafunctional habit control and topical lubricant on discomforts associated with burning mouth syndrome (BMS). Arch Gerontol Geriatr 2010;51:95-99.

12. Drage LA, Rogers RS 3rd. Burning mouth syndrome. Dermatol Clin 2003;21:135-145.

13. Isberg AM, Isacsson G, Williams WN, Loughner BA. Lingual numbness and speech articulation deviation associated with temporomandibular joint disk displacement. Oral Surg Oral Med Oral Pathol 1987;64:9-14.

14. Piagkou M, Demesticha T, Piagkos G, Georgios A, Panagiotis S. Lingual nerve entrapment in muscular and osseous structures. Int J Oral Sci 2010;2:181-189.

15. Alvira-González J, Gay-Escoda C. Sensory disturbances of buccal and lingual nerve by muscle compression: a case report and review of the literature. J Clin Exp Dent 2016;8:e93-e96.

16. Fricton JR, Kroening R, Haley D, Siegert R. Myofascial pain syndrome of the head and neck: a review of clinical characteristics of 164 patients. Oral Surg Oral Med Oral Pathol 1985;60:615-623.

17. Donnelly JM, Fernández-de-Las-Peñas C, Finnegan M, Freeman JL. Travell, Simons \& Simons' myofascial pain and dysfunction: the trigger point manual. 3rd ed. Philadelphia: Wolters Kluwer; 2019. pp. 268-293.

18. Hara S, Namekawa H. Adaptation of myofascial trigger point massage therapy for secondary burning mouth syndrome: a case report. Jpn J Orofac Pain 2011;4:63-70. 